\title{
Intelligent energy management of Microgrids with flexible demand response
}

\author{
Vishnu Suresh $^{1}$, Przemyslaw Janik ${ }^{1}$, Dominika Kaczorowska ${ }^{1}$ \\ ${ }^{1}$ Faculty of Electrical Engineering \\ Wroclaw University of Science and Technology \\ Wybrzeże Stanisława Wyspiańskiego 27, 50-370 Wrocław (Poland) \\ Phone/Fax number: +48 7132029 05, e-mail: vishnu.suresh@pwr.edu.pl ,przemyslaw.janik@ pwr.edu.pl
}

\begin{abstract}
This paper deals with the application of a mixed integer distributed ant colony optimization (MIDACO) algorithm as an energy management system for a microgrid represented by real time values and characteristics. The microgrid consisting of numerous sources of renewable energy such as solar photovoltaics, small hydro power plant and battery storage is also able to manage demand response in an intelligent manner so as to reduce the dependency on the utility grid during high peak conditions and also export power to the grid during lower load conditions.
\end{abstract}

Key words. Microgrids, MIDACO, Energy management system, Demand response, Renewable energy, Storage system

\section{Introduction}

The increasing population leading to an increasing energy demand along with the need to reduce the carbon footprint of modern power systems has led to focussed research in microgrids [1]. The microgrid is defined by the US Department of Energy as an entity with clear electrical boundaries consisting of distributed generation and interconnected loads. It behaves as a single entity with respect to the grid and is capable of operating in either a grid-connected mode via a point of common coupling or independently in an islanded mode[2].

The concept of energy management within microgrids is well-researched. Within the literature different approaches to energy management with regard to the optimisation algorithm used, demand response, modelling of microgrid components and different microgrid layouts are discussed as follows. A probabilistic approach to energy management consisting of forecasting of renewable energy resources and the load is carried out in [3]. A multi-objective optimisation reducing the capital costs, operating costs and net emissions is performed using Particle Swarm Optimisation (PSO). The components involved are solar PV, wind turbines, thermal power plant and battery storage. A similar yet different approach is presented in [4], here the optimisation algorithm used is the PSO but the microgrid layout is considerably different. It has 2 different microgrids consisting of different controllable loads, solar PV, storage systems, a hydro power plant, diesel generator and are connected to the utility via different buses. Moreover, the system is also connected to a wind park. The study does not utilise real time signals but the signals are modelled according to standard models available for individual microgrid components. PSO as an effective method for energy management in microgrids and virtual power plants has been further elaborated in $[5,6]$

In recent times there also has been an attempt to give more decision-making power to the consumer with regard to their production/consumption of power and are referred to as prosumers. [7] elaborates on this concept by explaining a residential hybrid microgrid. The objective function used in this case is minimization of the cost incurred by import of power from the grid, this is done by optimizing the charging and discharging of the storage system based on PV and load forecasts. [8] presents a novel optimization strategy based completely on demand response in-order to perform peak shaving operations. The model in this case consists of distributed generation, controllable loads, storage devices and has used an adjustable power margins for real time operation of microgrids.

The rest of the paper is divided into the following sections, 2 describes the microgrid layout of the study, 3 describes the mathematical model which forms the basis for energy management along with its algorithm and also explains MIDACO. 4 describes the results of the optimized microgrid and 5 enumerates the conclusions of the study.

\section{Microgrid Layout}

The Microgrid layout is shown in Fig. 1. There are numerous sources of renewable energy within the microgrid. It has 2 sources of solar photovoltaics of peak power capacity of $1.2 \mathrm{MW}$ and $1 \mathrm{MW}$. The data for the solar photovoltaics comes from the solar panels installed at Wroclaw University of Science and Technology at the Faculty of Electrical Engineering. The data has been multiplied by a suitable factor to represent the two sources but the characteristic of the curves remains the same as shown in Fig. 2. The values are measured at an interval of 10 minutes and the data presented here is for 4 days. 


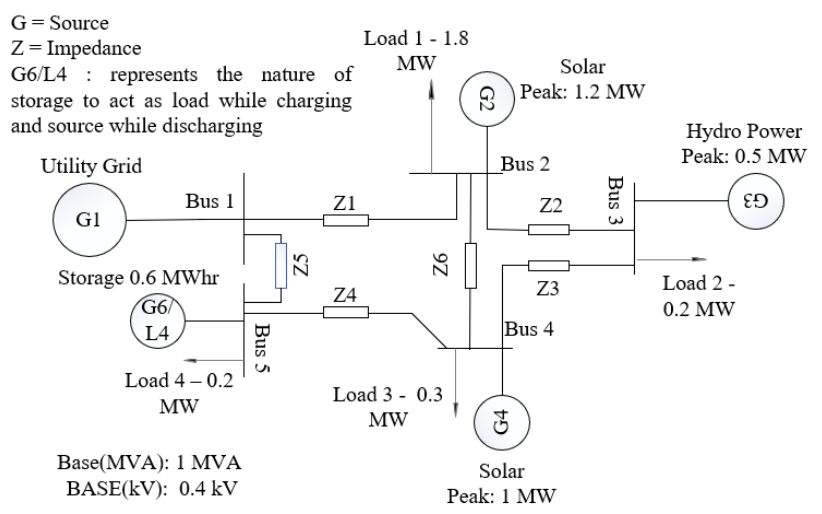

Fig. 1. Microgrid Layout

The length of 4 days is chosen so that the characteristic is clearly visible. The hydro power plant maintains a steady output of $0.5 \mathrm{MW}$, the battery storage used in the study has a capacity of $0.6 \mathrm{MWhr}$. The storage behaviour has been modelled in MATLAB according to [9] There are 4 loads within the system with variations throughout the day. The peak load values are mentioned in Fig 1. In load 1 the controllable load whose consumption can be shifted across a 24-hr cycle amounts to $200 \mathrm{~kW}$ at peak load demand. This is the net effect of all such loads within the system which could be washing machines, climate control of the house and water heaters. In case of load 3 it is $45 \mathrm{~kW}$, whereas for load 2 and load 4 is it $30 \mathrm{~kW}$.

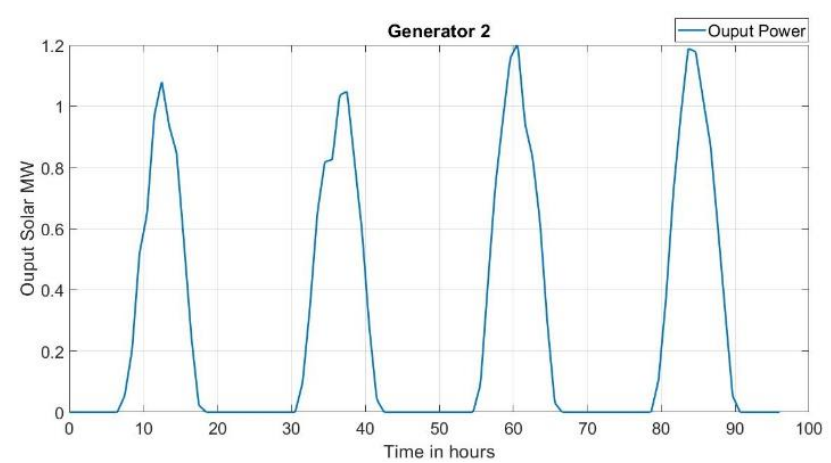

Fig. 2. Solar PV characteristic

The load characteristic is obtained from a village nearby the University and has a characteristic as shown in Fig 3. The other loads are modelled upon this characteristic.

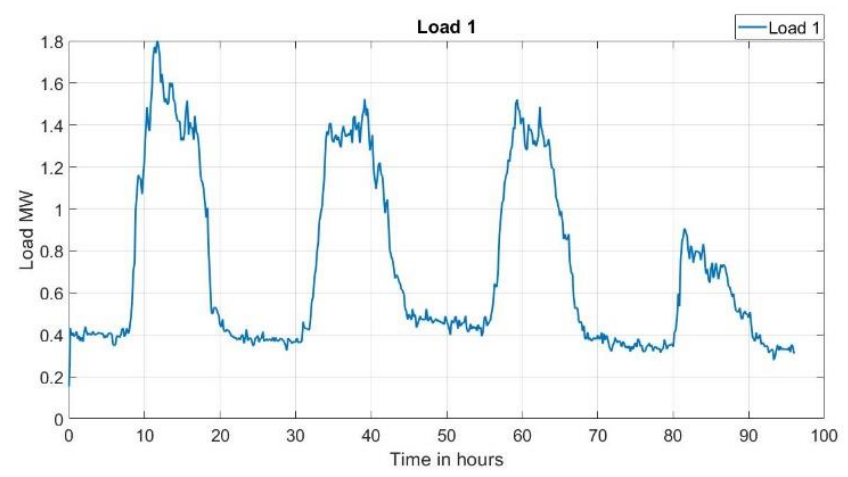

Fig. 3. Load characteristic

The values are measured at an interval of 10 minutes for a period of 4 days.
The distribution cables used are NKT LV IEC standard 60502-1:2004 lines, as shown in Table 1. It is an aluminium cable with XLPE insulation and PVC outer sheath having 1 core with $120 \mathrm{~mm}^{\wedge} 2$ cross-section. The energy storage device in this study is fictitious.

\section{Table I. - Cable Data}

\begin{tabular}{|l|c|c|c|}
\hline From & To & Distance $(\mathrm{m})$ & $r+j x$ \\
\hline Bus 1 & Bus 2 & 200 & $0.4740+0.1530 \mathrm{i}$ \\
\hline Bus 2 & Bus 3 & 110 & $0.2600+0.0840 \mathrm{i}$ \\
\hline Bus 3 & Bus 4 & 135 & $0.3200+0.1033 \mathrm{i}$ \\
\hline Bus 4 & Bus 5 & 215 & $0.5100+0.1640 \mathrm{i}$ \\
\hline Bus 5 & Bus 1 & 150 & $0.3558+0.1149 \mathrm{i}$ \\
\hline Bus 2 & Bus 4 & 250 & $0.5930+0.1914 \mathrm{i}$ \\
\hline
\end{tabular}

\section{Mathematical Foundation, MIDACO and Algorithm for energy management}

\section{A. Mathematical Foundation}

$$
\min J=\sum_{i=1}^{n_{g e n}}\left(P_{G}-P_{g i}\right)
$$

Subject to equality constraints

$$
\begin{aligned}
& P_{g i}-P_{d i}-P_{L}=0 \\
& Q_{g i}-Q_{d i}-Q_{L}=0
\end{aligned}
$$

Subject to inequality constraints

$$
\begin{aligned}
P_{g i(\text { minimum })} & \leq P_{g i} \leq P_{g i(\text { maximum })} \\
Q_{g i(\text { minimum })} & \leq Q_{g i} \leq Q_{\text {gi }(\text { maximum })} \\
V_{i(\text { minimum })} & \leq V_{i} \leq V_{i(\text { maximum })} \\
L_{i(\text { minimum })} & \leq L_{i} \leq L_{i(\text { maximum })} \\
B_{i(\text { minimum })} & \leq L_{i} \leq B_{i(\text { maximum })}
\end{aligned}
$$

Equation (1) represents the objective function, this study aims to keep the import of active power from the grid $\left(\mathrm{P}_{\mathrm{G}}\right)$ as low as possible thereby being self-sufficient and also reducing costs. (2) and (3) represent the equality constraints which are the power balance equations. $\mathrm{P}_{\mathrm{gi}}$ and $\mathrm{Q}_{\mathrm{gi}}$ are the active and reactive powers at bus $\mathrm{i}, \mathrm{P}_{\mathrm{di}}$ and $Q_{\mathrm{di}}$ are the active and reactive power demand at bus $\mathrm{i}$, $P_{L}$ and $Q_{L}$ are active and reactive power losses. $V_{i}$ represents the voltage values at $\mathrm{i}^{\text {th }}$ bus, $L_{i}$ represents the controllable load values at $\mathrm{i}^{\text {th }}$ bus, $B_{i}$ represents the battery storage output at $\mathrm{i}^{\text {th }}$ bus.

\section{B. MIDACO}

MIDACO is an extension of the ant colony optimization which is capable of handling both discrete and continuous variables [10]. It is based on the manner in which ants locate and utilise food sources. Food sources in this case being the various energy sources of the microgrid.

In MIDACO a construction graph is created wherein the vertices of the graph represent the food source (solution) and the edges of the graph determine the route taken by the ants to reach their food source. The food sources (solutions) located closer to the ants are used more 
frequently and are more attractive compared to the food sources (solution) located far away. In this way the ants select the optimal food source (solution). The path in this case represents the quality of the food source and higher the quality the shorter the distance. While the global minimum solution is achieved due to the construction graph and the movement of ants across it. Daemon actions make possible achieving local minimum objectives. When certain food sources are selected as optimum solutions, daemon actions (constraints) are applied to the solutions found and only those solutions which fit all the constraints are selected. The algorithm for the process is shown Fig 4. The performance of MIDACO in finding the global minimum solution especially in the case of optimal power flow applications has been documented in [11] which is accepted to be published in early 2020 and the work behind is done by the authors of this article.

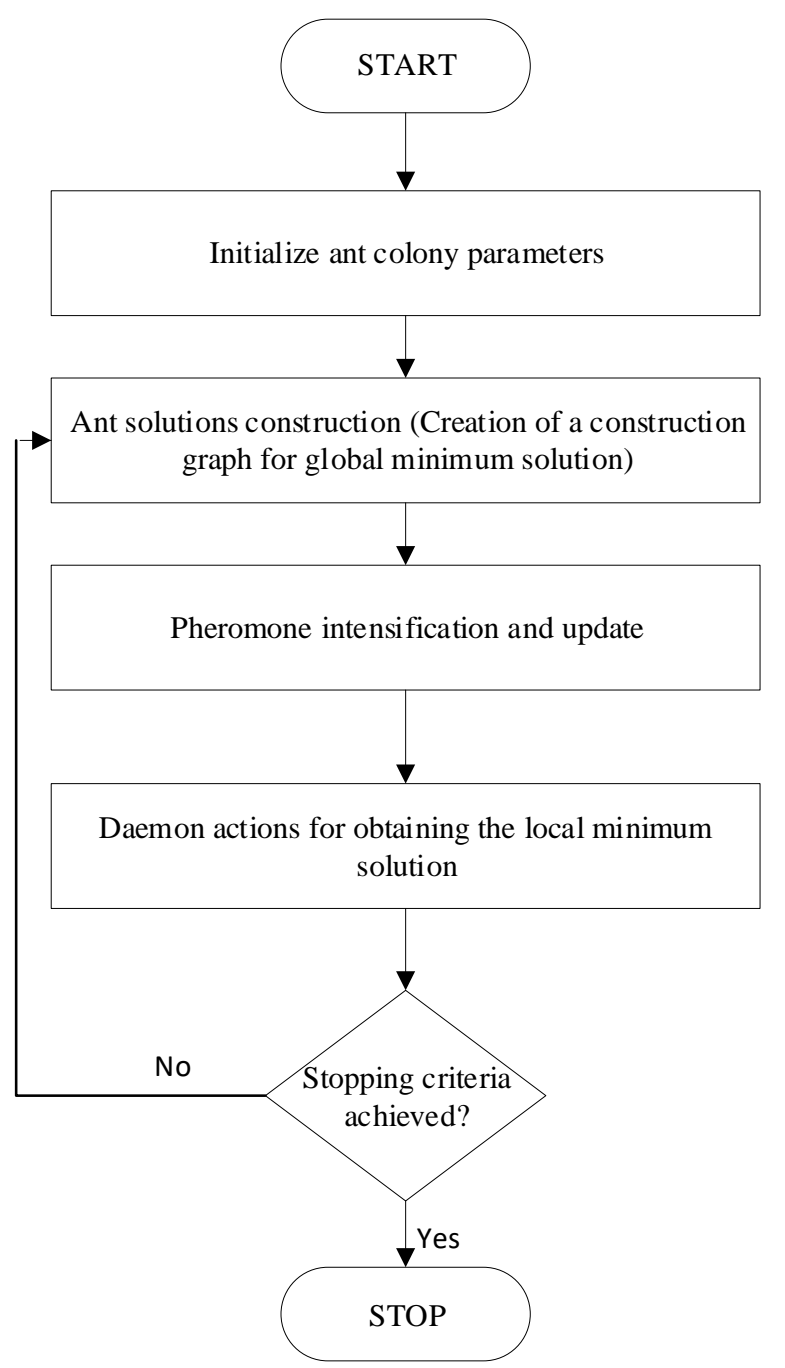

Fig. 4. Algorithm for MIDACO

\section{Algorithm for energy management}

The algorithm for energy management within the microgrid is shown in Fig. 6. It also includes the Battery Management System (BMS). The first priority is given to demand response. When the load demand within the microgrid exceeds the energy generated within the microgrid, the amount of load that can be shifted is calculated. For all the loads the controllable load amounts to about $15 \%$ of the load at its peak capacity. The load that is shifted is then later satisfied when the renewable energy generation within the microgrid exceeds the load demand. This ensures maximum utilisation of the energy produced within the microgrid. Moreover, the BMS also works in a similar manner. Once load shifting is performed, the excess load demand still within the microgrid is satisfied by the BMS. During times when there is excess energy within the microgrid and all pending loads are satisfied, the BMS enables charging of the storage system with the excess energy.

\section{Results}

Fig 5. represents the storage charging and discharging characteristics of the battery storage in both cases of demand response and no demand response. It can be noticed that the load shifting activity performed has significantly improved the characteristics of charging and discharging. There is no erratic charging and discharging observed because the corresponding erratic changes in load are smoothened by the load shifting.
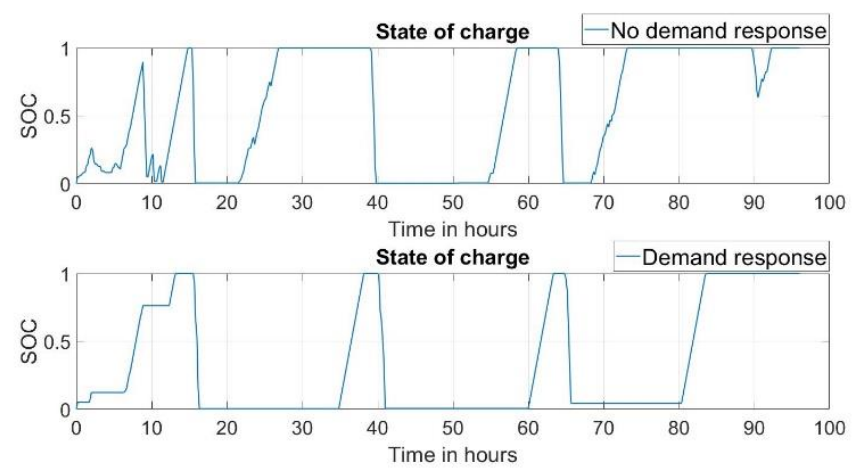

Fig. 5. Battery storage charging and discharging characteristics

Fig 7. depicts the load curves for cases when there is load shifting (demand response) and when there is no load shifting (no demand response) along with the total generated energy within the microgrid, due to the unique shape of the load curves used within this study. The results can be interpreted as follows. It can be seen that the part of the load curves which are smoothened are at the flat areas of the curve when the load demand is actually low. This is because even though the load is low in such regions these are the times when the load demand is actually higher than energy generated within the microgrid which is at its minimum which can be noticed from the total generation curve, hence the excess load that is shiftable is moved across to regions to when the energy produced is higher than the load demand. This is also the explanation for the spikes that are seen at the beginning of when the load demand actually starts to increase.

Fig 8. presents information on the exchange of power between the microgrid and the main grid via the point of common coupling. It can be inferred that with demand response the amount of power imported from the main grid is reduced. Also, it can be noticed that the power exported from the microgrid increases as overall the load demand fulfilled by the microgrid's own sources of energy is increased. 


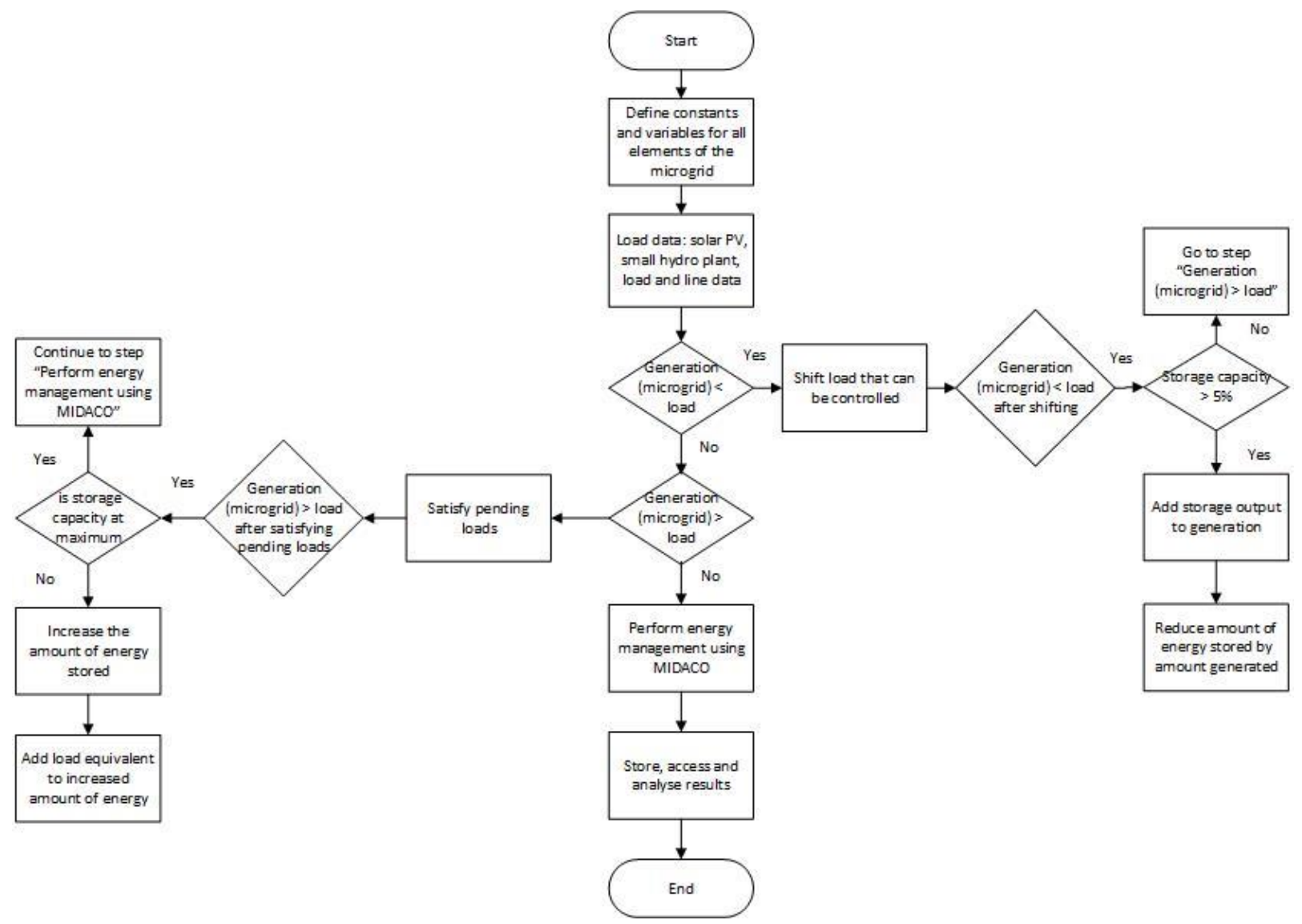

Fig. 6. Algorithm for energy management
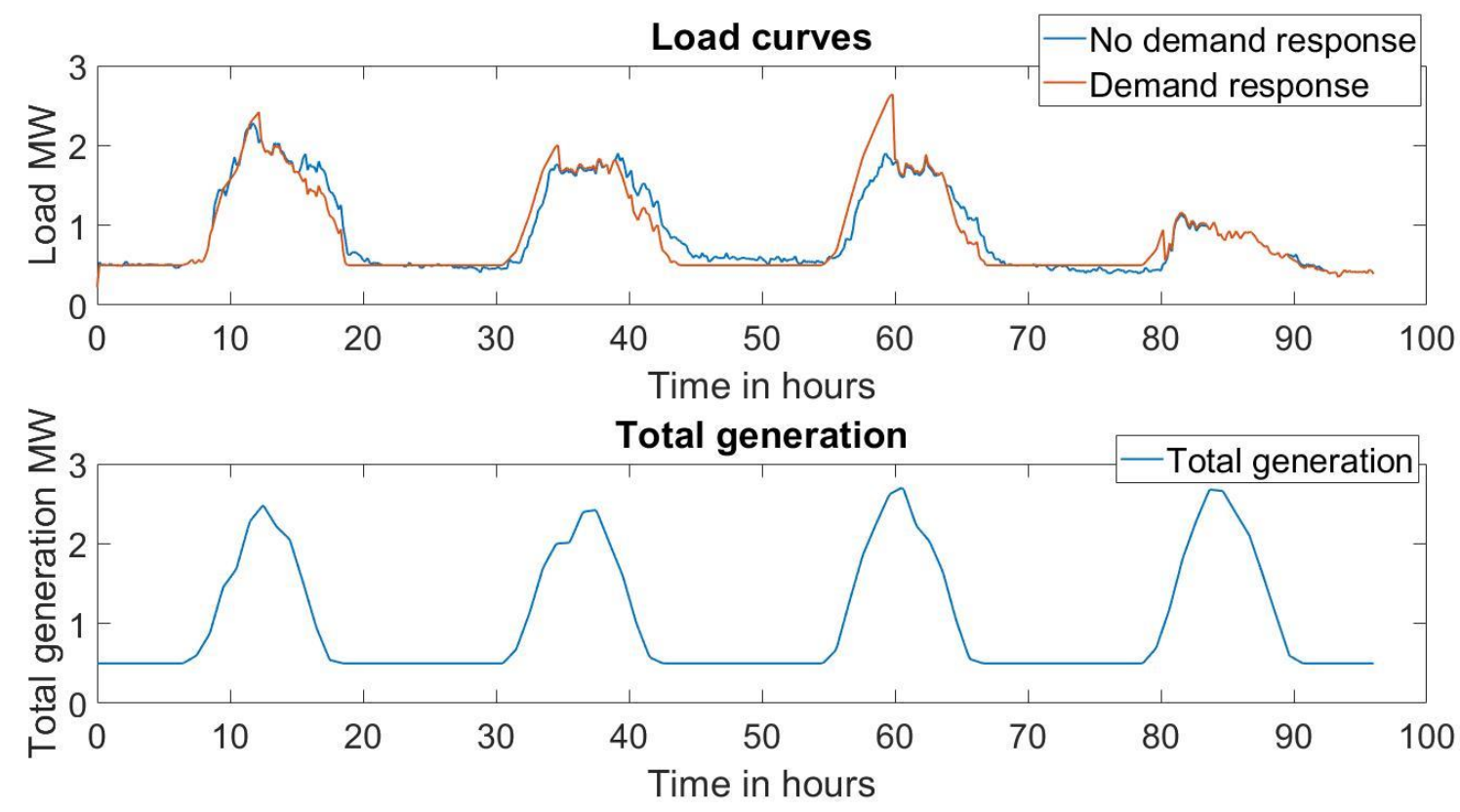

Fig. 7. Load shift and total microgrid generation

\section{Conclusions}

A deterministic energy management system for a microgrid with demand response was created with the use of a metaheuristic optimization algorithm. MIDACO was particularly chosen because of its suitability to optimal power flow applications. It was noticed that demand response can play a significant role in improving the life of storage systems due to better charging and discharging characteristics. It could lead to better utilisation of nondispatchable sources of energy within the microgrid and also improve export of power from the microgrid. While the objective function during this study was minimisation of import of power from the grid which was achieved it is believed that the true potential of MIDACO will be realised when cost minimisation or emission minimisation is set as the objective function which is planned as future work. It is also our understanding that implementation of 


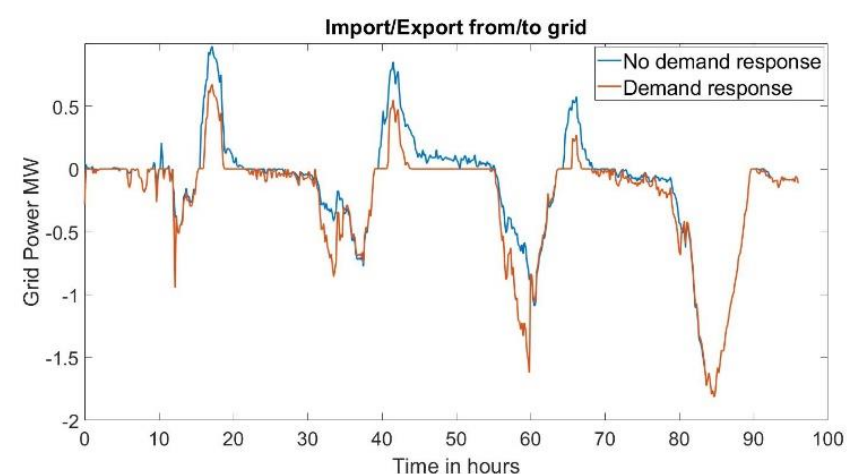

Fig. 8. Import/Export from/to grid

demand response as shown in this study can face numerous challenges in the real world due to the nature of contracts that has to be implemented between the distribution system operator and the consumer and also the willingness of the consumer to participate as well as follow the instructions provided by the system operator. Further future work involves selection of different optimization algorithms for energy management in order to select an appropriate one for the given system, to investigate the appropriate size of the storage unit for the system since it an apparatus that is planned to be installed at the facility and also to include models of electrical vehicle charging stations as additional loads.

\section{References}

[1] H. Fontenot, B. Dong, Modeling and control of buildingintegrated microgrids for optimal energy management A review, Appl. Energy. 254 (2019) 113689. doi:10.1016/j.apenergy.2019.113689.

[2] Office of Electricity Delivery and Energy Reliability, Smart Grid R \& D Program DOE Microgrid Workshop Report, (2011).
[3] A.Y. Saber, G.K. Venayagamoorthy, Smart micro-grid optimization with controllable loads using particle swarm optimization, IEEE Power Energy Soc. Gen. Meet. (2013) 1-5. doi:10.1109/PESMG.2013.6672780.

[4] E. Sortomme, M.A. El-Sharkawi, Optimal power flow for a system of microgrids with controllable loads and battery storage, 2009 IEEE/PES Power Syst. Conf. Expo. PSCE 2009. (2009) 1-5. doi:10.1109/PSCE.2009.4840050.

[5] D. Kaczorowska, J. Rezmer, Particle swarm algorithm for microgrid optimization, 2018 Innov. Mater. Technol. Electr. Eng. i-MITEL 2018. (2018) 1-4. doi:10.1109/IMITEL.2018.8370472.

[6] D. Kaczorowska, J. Rezmer, T. Sikorski, P. Janik, Application of PSO algorithms for VPP operation optimization, Renew. Energy Power Qual. J. 17 (2019) 91-96. doi:10.24084/repqj17.230.

[7] V.C.J. Sankar, M. Raghunath, M.G. Nair, Optimal scheduling and energy management of a residential hybrid microgrid, 2017 Innov. Power Adv. Comput. Technol. i-PACT 2017. 2017-Janua (2018) 1-6. doi:10.1109/IPACT.2017.8244988.

[8] L. Yunjia, M. Tenglong, L. Jialiang, Q. Zhanzhan, Independent microgrid day-ahead optimization based on demand response, Proc. 29th Chinese Control Decis. Conf. CCDC 2017. (2017) 5809-5814. doi:10.1109/CCDC.2017.7978205.

[9] Y. Levron, J.M. Guerrero, Y. Beck, Optimal power flow in microgrids with energy storage, IEEE Trans. Power $\begin{array}{llll}\text { Syst. } & 28 & \text { (2013) 3226-3234 }\end{array}$ doi:10.1109/TPWRS.2013.2245925.

[10] M. Schlüter, J.A. Egea, J.R. Banga, Extended ant colony optimization for non-convex mixed integer nonlinear programming, Comput. Oper. Res. 36 (2009) 22172229. doi:10.1016/j.cor.2008.08.015.

[11] V. SURESH, M. Janik, P. , Jasinski, Metaheuristic Approach to Optimal Power Flow Using Mixed Integer Distributed Ant Colony Optimization Metaheuristic Approach to Optimal Power Flow Using Mixed Integer Distributed Ant Colony Optimization, Arch. Electr. Eng. Pol. Akad. Nauk. (n.d.). 